\title{
Diarios de información económica: la diferencia existe
}

\author{
Por Javier Peralta \\ Como lo afirma el siguiente artículo, no todos
} los diarios económicos exhiben la misma calidad informativa. Estas distinciones, de carácter cuantitativo y cualitativo, son abordadas en esta investigación desde el punto de vista del producto esencial del reporteo: la noticia. Del análisis efectuado con artículos de diarios chilenos y extranjeros surgen interesantes conclusiones, que pueden llevar a decisiones concretas en muchos medios.

U $\mathrm{n}$ un estudio sobre medios escritos realizado en Estados Unidos, Dan Berkowitz y Douglas Beach², llegaron a la siguiente conclusión: "Las fuentes son las que modelan la noticia, más que el periodista que la escribe". Según ellos, los periodistas se han ido acostumbrando a depender de un cierto tipo de fuentes, especialmente de las rutinarias, para escribir sus artículos. Esto los ha ido alejando de una búsqueda de nuevas fuentes, de nuevas historias y de los cambios en los intereses del público.

Este tipo de estudio se ha ido propagando en Estados Unidos, tras el objetivo de establecer cómo influye la cantidad y variedad de fuentes en los artículos periodísticos. Sin embargo, en Chile es poco lo que se ha avanzado en la materia. Se cree que el diagnóstico respecto de la dependencia de los periodistas chilenos de fuentes rutinarias es similar al de Estados Unidos, pero no se han realizado estudios acabados.

El presente análisis de fuentes en el periodismo económico pretende dilucidar en cierta medida alguna idea de lo que sucede en nuestro país. Siguiendo los modelos de los estudios realizados en Estados Unidos, el objeto de este trabajo es establecer qué origen tienen las noticias en el periodismo económico, qué tipo y qué variedad de fuentes se utilizan, cuáles son sus características, y si de alguna manera estos aspectos influyen en la calidad de la noticia. A su vez, y para someter los resultados a un análisis comparativo, fueron seleccionados algunos diarios extranjeros, con el fin de establecer cómo trabajan ellos con las fuentes, y en qué se asemejan y en qué difieren del periodismo económico chileno.

'Este estudio formó parte del curso "Periodismo Económico", dictado por el profesor Patricio Bernedo. El autor agradece al profesor Paulo Ramírez por su guía en la planificación general y elaboración de este trabajo.

"Dan Berkovitz y Douglas W. Beach, "News sources and context: effect of routine news, conflict and proximity", en Journalism Quarterly, Spring 1993, vol. $70 \mathrm{~N}^{\circ} 1$, pp. 508-513. 
No se busca dar cuenta de todo lo que sucede en nuestro periodismo económico, sino de las líneas generales que sigue y de la situación chilena respecto del periodismo en otros países.

La utilidad de este análisis está dada por la comprobación o rechazo de las hipótesis de trabajo. Los resultados podrán servir en la determinación del nivel de desarrollo de nuestro periodismo: si hay dependencia respecto de las fuentes, si éstas son poco variadas, si el fenómeno afecta la calidad de la noticia, si hay diferencias entre Chile y el extranjero. En un momento en que el periodismo económico chileno está buscando formas de adaptarse a lo que quiere el público, en que ha mostrado signos de desorientación, en que medios como $\mathrm{El}$ Mercurio y El Diario han pasado por procesos de reformulación, este análisis puede servir para aclarar algunos puntos y dar ciertas pautas, al menos respecto de la utilización de fuentes y su relación con la noticia. Estos dos elementos, como afirman los autores recién citados, pueden estar estrechamente relacionados.

\section{Hipótesis de trabajo}

Como ya se ha dicho, sobre la base de estudios anteriores se han deducido varias conclusiones acerca del funcionamiento del periodismo en general respecto de las fuentes en Estados Unidos. El objetivo principal de este análisis es observar qué ocurre con el periodismo económico en nuestro país.

Una primera conclusión extraída de los resultados obtenidos en Estados Unidos ${ }^{3}$ es que existe una alta dependencia de parte de los periodistas de los canales rutinarios. También agregan que este tipo de fuentes afecta la variedad de noticias. Para efectos de este análisis, lo primero que se hará es comprobar este diagnóstico en el periodismo económico específicamente. Se plantearán las siguientes hipótesis:

Hipótesis 1: Existe una relación directa entre el origen de la noticia (canal informativo), la cantidad y variedad de fuentes y la calidad de la noticia. Si en el periodismo económico se recurre con excesiva frecuencia a las fuentes rutinarias, se verá afectada la variedad de fuentes y se entregará una información de baja calidad.

De comprobarse esta hipótesis, se pueden aventurar algunas de sus posibles consecuencias. En especial se hace referencia a la preocupación particular de un grupo de investigadores, Brown y otros ${ }^{4}$, que argumentan sobre cómo el mal uso de las fuentes actúa en detrimento del rol de la prensa en una sociedad democrática.

Una segunda hipótesis de trabajo dice relación con la comparación entre los diarios, tanto chilenos como extranjeros. Siempre se tiene el prejuicio de que el periodismo chileno deja mucho que desear al enfrentárselo con el de Europa y el de Estados Unidos. No se puede hablar de una manera general, pero sí analizarlo de la siguiente forma particular:

Hipótesis 2: La situación descrita en la hipótesis 1, en relación a la alta dependencia respecto de fuentes rutinarias, no es distinta entre los medios chilenos, sin embargo presenta una franca y clara desventaja respecto

Tal como muchas investigaciones realizadas en Estados Unidos, este estudio llega a una nítida conclusión: existe una relación directa entre los métodos de reporteo utilizados por los periodistas y la calidad informativa de las noticias que ellos publican.

\footnotetext{
${ }^{3}$ Dan Berkovitz y Douglas W. Beach, op. cit.; Jane Delano Brown, et al, "Invisible Power: Newspaper News Sources and the Limits of Diversity", en

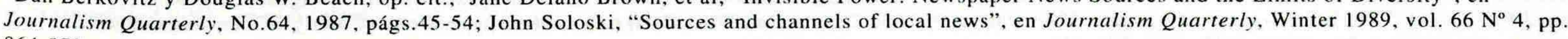
864-870.

4 Jane Delano Brown, et al., op. cit.
} 


\section{En los medios chilenos analizados se depende} excesivamente de los canales informativos rutinarios, es decir, aquellos en los que la fuente tiene la iniciativa primordial y puede modelar casi a su antojo el producto informativo.

de los medios extranjeros. Se comparará la situación entre tres diarios chilenos respecto de tres medios extranjeros.

Una tercera hipótesis apunta a determinar cómo la existencia de reporteo adicional (esto es, de una actitud activa de parte de los reporteros que no se limita a presenciar un hecho y transcribirlo, sino que indaga sobre circunstancias ligadas al acontecimiento) también afecta la cantidad de fuentes y la calidad de la noticia:

Hipótesis 3: Existe una estrecha relación entre el reporteo adicional, la cantidad y variedad de fuentes y la calidad de la noticia. La ausencia de este elemento se traduce en noticias con escasas fuentes y de contenido de baja calidad.

Inmediatamente se plantea la cuarta hipótesis:

Hipótesis 4: La situación descrita en la hipótesis 3 , en relación a la influencia del reporteo adicional sobre las fuentes y la calidad informativa, es más notoria en el periodismo nacional que en el extranjero, ya que en nuestro país el reporteo adicional es menos frecuente.

Estas cuatro hipótesis pretenden demostrar que en el periodismo económico existe un problema, que éste radica en el origen de la noticia y en el escaso reporteo, que se manifiesta en el bajo número de fuentes y mala calidad de las noticias, y que este problema es más frecuente entre los medios chilenos que los extranjeros.

\section{Metodología}

\section{1.- Técnica de muestreo: selección de medios, fechas y noticias}

De acuerdo a los objetivos de este análisis y a los recursos de que se disponía, se decidió analizar tres diarios chilenos y tres diarios extranjeros. El criterio utilizado para seleccionar los medios fue el de trabajar sólo con aquellos que privilegian la información económica. De esta manera, en Chile se seleccionaron los diarios Estrategia, El Diario ${ }^{5}$ y El Mercurio (cuerpo B "Economía y Negocios"). Si bien este último medio no da prioridad especial a la economía, la existencia de un cuerpo especial dedicado al tema lo hace comparable con la información de los otros dos diarios elegidos, que son principalmente económicos.

En cuanto a los diarios de fuera del país, se decidió trabajar con un número igual a la cantidad de medios chilenos. Para obtener algunos resultados de la comparación, se seleccionaron medios de tres países distintos, representativos de tres zonas geográficas diferentes. Se eligieron aquellos que priorizan la información económica. Se incluyó en la muestra al diario argentino Ambito Financiero, por ser un diario de un país sudamericano, con una realidad cultural semejante a la nuestra, y por ser uno de los más importantes en el área económica en Argentina. La utilidad de tener un diario argentino en la muestra está dada por esta misma cercanía geográfica y cultural: se puede establecer qué tipos de diferencias existen en el tratamiento de noticias y de fuentes entre diarios chilenos y el más importante diario económico argentino.

El segundo medio extranjero elegido fue el norteamericano The Wall Street Journal, el diario de mayor venta en Estados Unidos (1 millón 800 mil ejemplares al

${ }^{5}$ Debe tomarse en consideración que El Diario no había sufrido aún las modificaciones de su reestructuración, aparecidas el 7 de agosto de 1995.

${ }^{6}$ Daniel Riffe, et al, "The Effectiveness of Random, Consecutive Day and Constructed Week Sampling in Newspaper Content Analysis", en Journalism Quarterly, Vol 70 No.1, Primavera 1993, págs. 133-139. 
día), cuya reputación como diario económico es conocida, y que constituye un excelente representante del periodismo norteamericano. En estas mismas características radica la utilidad de tenerlo en la muestra para fines comparativos. El último diario incluido fue el Financial Times de Londres. También muy buen representante del periodismo económico y uno de los diarios más importantes de Europa.

En cuanto a la selección de noticias, para que la comparación fuera válida y pudieran obtenerse parámetros lo más objetivos posibles, se utilizó un criterio de selección que tendiera a homogeneizar las noticias escogidas, dentro de las limitantes naturales dadas por la diferencia de los medios. Se decidió tomar cinco noticias por cada diario en cada fecha analizada. Se consideró este número como una cantidad suficiente para hacer el estudio, en los márgenes dados por el tiempo y los recursos disponibles.

Las noticias fueron elegidas aleatoriamente. Sólo podían ser seleccionadas aquellas noticias que fueran económicas (la mayoría de los diarios cubre otros sectores además de economía), reporteadas por personal del diario, y locales (de cada país). Para eliminar aquellas noticias demasiado cortas, sólo se consideró en la selección la más destacada e importante de cada página, considerando que ésas son, de acuerdo al diario, las de mayor relevancia y a las que se les ha dedicado más tiempo y más recursos.

Con esta selección se dejaron de lado factores que podían desequilibrar la balanza en perjuicio de los medios más chicos, o aquellos elementos sobre los que el diario no tiene mayor actuación, como son las noticias de agencias, los cables, las reproducciones de otras publicaciones, las noticias de corresponsales, etc.
El proceso de selección de fechas fue elegido entre varios métodos que se han utilizado para este tipo de muestreos. Siguiendo a Riffe y otros 6 y siendo éste un análisis general, no sobre un tema específico, y que cubre varios medios (no uno solamente, como otros estudios), se eligió el método de "semana construida"7.

La "semana construida" consiste en revisar los medios seleccionados durante el período de una semana. Sin embargo, no de días consecutivos, sino de días tomados de distintas semanas representativas de el período en estudio. Así, se escoge aleatoriamente el lunes de una semana determinada, el viernes de la semana siguiente, etc. Quedan todos los días representados, lo que puede no suceder en las muestras de selección simple, y logra superar dos problemas:

- asume la variación cíclica que pueden tener los diarios de una semana a otra, ya que hay semanas más ricas en historias que otras;

- se soluciona el problema de las diferencias en contenidos entre los distintos días de la semana.

En este caso se seleccionó el período comprendido entre el 17 de abril y el 17 de mayo de 1995. De esas semanas se eligieron los días al azar, quedando las siguientes fechas seleccionadas: lunes 17 de abril, viernes 28 de abril, jueves 4 de mayo, martes 9 de mayo, miércoles 17 de mayo. Sólo se consideraron los días

$$
\text { Por lo general, la cantidad de fuentes utilizadas }
$$
por los diarios económicos chilenos es muy baja: apenas un poco más de una fuente como promedio. La noticia de fuente única es la que prima, generando como consecuencia una baja calidad de la información.

\footnotetext{
7 "Constructed week" en el original.
} 
Además de la escasez de fuentes, la diversidad de esas fuentes es muy baja, lo que disminuye sus aportes informativos, por cuanto representan muy pocos puntos de vista frente a los hechos abordados.

porque varios de estos diarios no se publican los fines de semana.

Dos salvedades metodológicas respecto de la selección de fechas: el lunes 17 de abril de 1995, el diario Financial Times no salió por ser feriado en Gran Bretaña. En su reemplazo se tomó el ejemplar del fin de semana inmediatamente anterior, del 15/16 de abril. Este cambio no representa grandes modificaciones, porque la característica del día lunes está dada por la publicación de reportajes en profundidad, elemento que se repite en las ediciones del fin de semana. La segunda observación es respecto del ejemplar de The Wall Street Journal del día viernes 28 de abril. Ese día, el diario no llegó a Chile, ni a la venta directa ni a bibliotecas, por lo que fue reemplazado por el del viernes siguiente, 5 de mayo.

\section{2.- Ficha de trabajo}

Este fue el modelo utilizado para registrar la información de cada noticia:

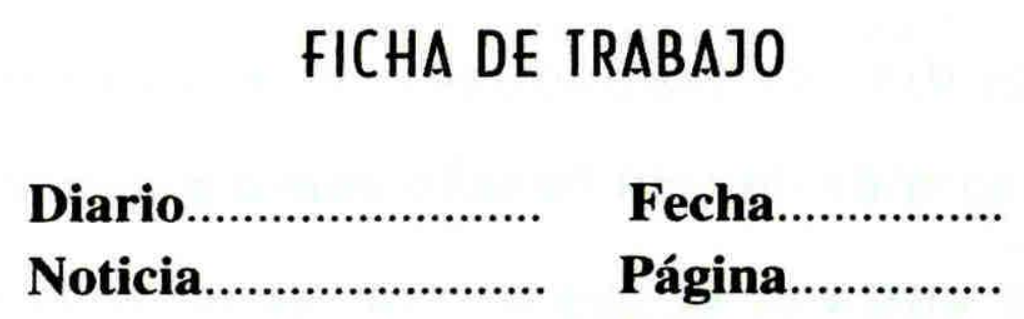

1.- Número de Fuentes

\section{2.- Canal de información}

Rutinario Informal

\section{3.- Reporteo Adicional}

Sí o No

\section{4.- Tipo de fuente}

Gbno.

Afiliada

Gbno. Ec.

Asociada

Indep.

Afiliada Ec.

Asociada Ec.

Indep. Ec.

\section{5.- Nivel de la fuente}

Ejecutivo Vocero

Funcionario o trabajador No especif.

\section{6.- Atribución de fuentes}

- Con atribución: completa incompleta anónima

- Sin atribución

\section{7.- Indicadores de calidad}

- Instrucción:

- Proceso:

- Antecedentes:

- Consecuencias:

- Pros y contras:

- Intereses actores:
SI, NO, N/C

SI, NO, N/C

SI, NO, N/C

SI, NO, N/C

SI, NO, N/C

SI, NO, N/C 


\section{1.- Explicación de la ficha de trabajo}

En primer lugar, se identifica en forma precisa cada una de las noticias: el medio, la fecha, el título de la noticia y la página. Esto permite remitirse a la crónica específica en caso de necesidad o revisión.

\section{a.- Número de fuentes}

Establece la cantidad de fuentes a la que recurrió el periodista al escribir su nota. Para efectos de este análisis, sólo se considera como fuentes aquellas personas citadas directa o indirectamente en la noticia. La descripción de cuánto ha caído la bolsa, o el precio de cotización del dólar, por ejemplo, son hechos: si en las notas en que se los describe no hay persona citada, aparecerá como una noticia sin fuentes. De esta manera, la cantidad de fuentes puede no ser siempre determinante en una noticia, aunque no deja de ser una variable de importancia.

\section{b.- Canal de información}

Esta categoría se estableció de acuerdo al estudio realizado por Leon V. Sigal ${ }^{8}$. El autor clasifica el origen de la noticia en tres canales distintos: rutinario, informal e iniciativa del medio. Sigal entiende canales como las vías por las cuales la noticia llega al reportero.

- Los canales rutinarios son: a) procedimientos oficiales (como juicios, audiencias, elecciones, etc); b) comunicados de prensa; c) conferencias de prensa y d) eventos no espontáneos, como discursos, congresos, seminarios, desfiles, inauguraciones, etc.

- Canales informales son: a) informes de empresas; b) filtración de información; c) procedimientos no gubernamentales, que corresponden a todos los eventos programados de empresas y asociaciones y d) publicaciones de otros medios.

- La iniciativa del medio corresponde a: a) entrevistas que hace el medio; b) eventos espontáneos que reportea el periodista; c) investigación independiente y d) análisis y conclusiones del propio periodista.

Sigal hace la aclaración de que muchos de los canales informales puede ser tomados como rutinarios, en cuanto son procedimientos no espontáneos cuya única diferencia es que son organizados por la empresa privada o por asociaciones en vez del gobierno. La clasificación del autor es de 1973. Para que estas categorías sean funcionales al presente análisis, se ha interpretado como rutinarias todas las conferencias y comunicados de prensa que emiten las empresas, compañías y asociaciones. Estas no quedan bien clasificadas en el esquema de Sigal, porque no eran tan frecuentes ni revestían gran importancia hace 20 años. Quedan clasificados como informales todos los eventos organizados por estas instituciones de los que se desprenden noticias (reuniones, congresos, manifestaciones, etc.)

Este punto es el clave para poder comprobar la veracidad de la afirmación contenida en las hipótesis de trabajo 1 y 2 .

\section{c.- Reporteo adicional}

Este punto pretende establecer la preocupación del periodista por reportear más allá del hecho mismo que originó la noticia. Por ejemplo, en caso de una conferencia de prensa, si el periodista recaba más información respecto de los antecedentes, de los pros y contras del suceso anunciado. Se pretende demostrar que esta

El estudio muestra la existencia de muy poca iniciativa de los diarios chilenos en el momento de definir los hechos de la realidad a los que destinará recursos de cobertura. $Y$, como ha sido demostrado, una menor iniciativa redunda en una menor calidad informativa.

${ }^{8}$ Sigal, Leon V., Reporters and Officials: The Organization and Politics of Newsmaking, D.C. Heath and Company, Lexington, 1973 , págs. 119-130. 
Una buena alternativa para superar las limitaciones derivadas de la falta de iniciativa propia es recurrir al reporteo adicional en las noticias surgidas como producto del canal rutinario. Con ese reporteo adicional es posible incrementar significativamente la calidad de la información publicada.

categoría es determinante en lo que ha sido llamado calidad de la noticia, a través de la hipótesis 3 .

\section{d.- Tipo de fuente}

Esta categoría es reconocida por los autores de este tipo de estudios ${ }^{9}$. Se diferencian aquí las fuentes de gobierno (como el Ministerio de Economía o Hacienda, el Banco Central, otros ministerios, y los otros poderes e instituciones), las afiliadas (empresas privadas, organizaciones no gubernamentales, etc), las asociadas (las asociaciones) y las independientes. Aquí también se establece la diferencia entre las que son económicas (aquellas relacionadas directamente con el sistema económico) y las no económicas.

\section{e.- Nivel de la fuente}

Establece quién es el que habla: un ejecutivo, un vocero, un trabajador o funcionario, o si no está identificado. Para algunos autores ${ }^{10}$, la diferencia entre los que hablan es determinante en la credibilidad de la noticia. Este punto no va a ser profundizado mayormente en esta investigación, aunque el material siempre podrá ser útil para futuros análisis.

\section{f.- Atribución de la fuente}

Este elemento ${ }^{11}$ establece si el periodista identifica claramente a quién se está refiriendo, o a quién está citando. Si le da nombre, cargo e institución a la que pertenece, es atribución completa. Si omite alguno de estos elementos, es incompleta. Si aclara que la fuente mantiene su anonimato por petición expresa, se clasifica como fuente anónima. Se llama sin atribución, cuando se da una información y no se dice de donde viene (ejemplo, "se comenta que...", "circula el rumor...", etc.).

Este punto es de vital importancia en dos ámbitos. Si se recurre mucho a la fuente sin atribución o a la fuente anónima, la credibilidad de un medio se puede ver afectada. Por otra parte, Brown dice que es deber de la prensa dejar en claro de dónde vienen las declaraciones y afirmaciones que publica, por ser una responsabilidad que asume el medio ante el público en un sistema democrático. Sin embargo, para efectos de este análisis particular, este elemento no va a ser utilizado, pero puede ser útil para interpretar los datos desde perspectivas no consideradas en estas hipótesis de trabajo.

\section{g.- Indicadores de Calidad}

Este último punto es clave en el desarrollo de la investigación; sirve para comprobar todas las hipótesis de trabajo que se manejan en este estudio.

Para la elaboración de esta clasificación se recurrió al planteamiento de Doris Graber ${ }^{12}$. Se le hicieron algunos ajustes para adecuarlo al objeto de estudio, ya que la autora estableció estos indicadores para noticias políticas y en Estados Unidos.

Son varios los mecanismos que existen para medir la calidad de la noticia ${ }^{13}$. Sin embargo, estos seis

\footnotetext{
${ }^{9}$ Especialmente por Dan Berkowitz y Douglas W. Beach, op. cit.

${ }^{10}$ Brown et al, op. cit.

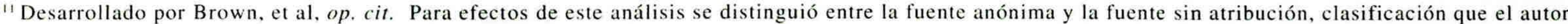
citado no hace.

${ }^{12}$ Citada por Richard Hofsetter y David Dozier en "Useful News, Sensational News: Quality, Sensationalism, and Local TV News", en Journalism Quarterly, Vol.63, No.4, Invierno 1986.

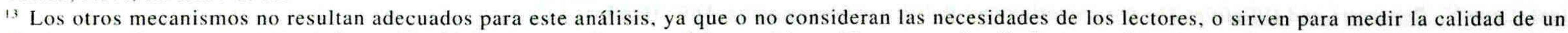
diario específico, como el diseñado por David Coulson en "Impact of Ownership on Newspaper Quality”, Journalism Quarterly, Vol.71, No.2, verano 1994, págs.403-411.
} 
elementos son especialmente importantes porque miden el nivel informativo de la noticia, permiten hacer un análisis más global y general desde el punto de vista temático, y constituye una evaluación de la utilidad que tiene la crónica para el público, que es el destinatario final del producto. Otras mediciones se hacen en virtud de las características formales o de las fuentes. Los parámetros de medición son:

- Instrucción: algunos temas tratados por las noticias pueden tener instrucciones precisas para el público, que le sirven en cuanto la noticia le afecta directamente. Por ejemplo, si se anuncia un reajuste de pensiones, se indica qué impacto tendrá para distintos tramos de ingreso, se menciona cuándo comienza a regir, etc.

- Proceso: en la mayoría de los casos, la noticia hace alusión de manera tácita al proceso en que está inserto el hecho. Sin embargo, este proceso generalmente no está explicado y puede ser desconocido por el público. (Por ejemplo, si se anuncia que la Contraloría aún no despacha las tarifas telefónicas, se debe explicar por qué es la Contraloría la que despacha dichas tarifas).

- Antecedentes: este rasgo está presente en la mayoría de las noticias. Es la explicación de los hechos anteriores que determinan el suceso noticioso.

- Consecuencias: es uno de los elementos claves de la noticia. Es el "en qué se traduce el hecho publicado".

- Pros y contras: son los argumentos a favor y en contra del hecho. Las dos caras de la moneda.

- Intereses actores: algunas noticias explican claramente qué tipos de intereses se ponen en juego, quiénes se verían afectados y quiénes favorecidos.

\section{Interpretación de los resultados 14}

Antes de comenzar el análisis de las estadísticas es preciso aclarar que en muy pocas ocasiones se entrará en el detalle de cada medio, salvo donde sea pertinente. La primera tabla muestra el promedio de fuentes por noticia en cada medio.

\section{TABLA No. 1}

\section{Promedio de fuentes por noticia}

Total noticias: 150

Total fuentes: 336

Promedio de fuentes por noticia: 2,24

\begin{tabular}{|lr} 
& Según medio \\
\hline Estrat. & 1,1 \\
Mercurio & 1,1 \\
El Diario & 1,5 \\
Ambito & 1,8 \\
Wall Street & 5,7 \\
F.Times & 2,2 \\
\hline
\end{tabular}

Los resultados son bastante decidores. Los medios chilenos registran la menor cantidad promedio de fuentes, más bajos que el promedio general. Esta situación no es muy distinta para Ambito Financiero, aunque está más cerca del promedio. Financial Times queda justo en el límite. Sin embargo se observa que el Wall Street Journal es el que eleva drásticamente la vara de medición, demostrando su mayor utilización de fuentes.

Si se hace el ejercicio de excluir del conteo al Wall Street Journal, el total desciende a 195 fuentes en 125 noticias. Esto reduce el promedio de fuentes por noticia a 1,56. Esto demuestra que los diarios chilenos y el argentino están sumamente lejos de compararse con el Wall Street Journal, y, peor aún, los tres medios de nuestro país quedaron bajo el promedio aún sin incluir a ese diario en la medición.

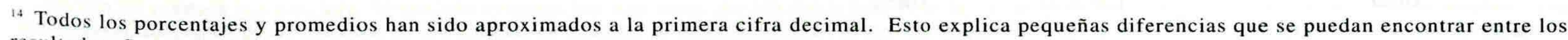
resultados. Son variaciones insignificantes, que de nonguna manera afectan los resultados de la investigación.
} 
La tabla 1.1 muestra los resultados más útiles para efectos de este análisis: el promedio de fuentes de los diarios chilenos en comparación con los diarios extranjeros.

TABLA No.1.1

\begin{tabular}{|c|c|c|c|}
\hline \multicolumn{4}{|c|}{$\begin{array}{l}\text { Promedio de fuentes por noticia } \\
\text { según nacionalidad }\end{array}$} \\
\hline & & Extranjero & Total \\
\hline Noticias & 75 & 75 & 150 \\
\hline Fuentes & 91 & 245 & 336 \\
\hline Promedio & 1,2 & 3,3 & 2,24 \\
\hline
\end{tabular}

Para extraer conclusiones con algún grado de validez no bastan estos resultados. Se hace necesario e interesante entrar en el detalle de la comprobación de las hipótesis.

\section{Comprobación Hipótesis 1}

El siguiente cuadro muestra las estadísticas respecto del origen de la noticia, tanto la cantidad como el porcentaje total que corresponde a cada canal de información.

\section{IABLA No.2}

Origen de la noticia (canal de información)

\begin{tabular}{|c|c|c|}
\hline & Total & Porcentaje \\
\hline Rutinario & 74 & $49,3 \%$ \\
\hline Informal & 36 & $24 \%$ \\
\hline \multicolumn{3}{|l|}{ Iniciativa } \\
\hline medio & 40 & $26,7 \%$ \\
\hline Total & 150 & $100 \%$ \\
\hline
\end{tabular}

En los resultados generales se comprueba la primera parte de la hipótesis 1: existe mayor dependencia de los medios de los canales de información rutinarios. Sin embargo, al mirar el detalle, vemos que algunos medios escapan a esta tendencia (ver tabla 6.1, en nota 12).

Para entrar de lleno a la comprobación de esta hipótesis es preciso observar la siguiente tabla:

IABLA No.3

\begin{tabular}{lccc}
\multicolumn{4}{|c}{$\begin{array}{c}\text { Promedio de fuentes por noticia según canal } \\
\text { informativo }\end{array}$} \\
& Noticias & Fuentes & Promedio \\
& & & \\
& 74 & 117 & 1,6 \\
Rutinario & 136 & 97 & 2,7 \\
$\begin{array}{l}\text { Informa } \\
\text { Iniciativa }\end{array}$ & 40 & 122 & 3,1 \\
medio & 450 & 336 & 2,24 \\
& & & \\
Total & 150 &
\end{tabular}

Aquí se demuestra una de las relaciones entre variables que se plantea en la primera hipótesis: el canal informativo influye directamente en la cantidad de fuentes por noticia. Se observa que el promedio obtenido para las noticias de origen rutinario es más bajo que el promedio general, y no sólo eso, es el único inferior a esta cifra. Esto queda confirmado en la tabla 3,1, donde se registra que sólo un $12 \%$ de las noticias rutinarias superan el promedio general. 
IABLA No.3.1

\begin{tabular}{|c|c|c|c|}
\hline \multicolumn{4}{|c|}{$\begin{array}{l}\text { Noticias con dos o más fuentes y con tres } \\
\text { según canal informativo } \\
\text { (porcentajes) }\end{array}$} \\
\hline & $\mathrm{N}^{\circ}$ & con 2 o más & con 3 o más \\
\hline Rutinario & 74 & $36,5 \%$ & $12,2 \%$ \\
\hline Informal & 36 & $66,7 \%$ & $44,4 \%$ \\
\hline \multicolumn{4}{|l|}{ Iniciativa } \\
\hline medio & 40 & $55,0 \%$ & $37,5 \%$ \\
\hline Total & 150 & $48,7 \%$ & $26,7 \%$ \\
\hline
\end{tabular}

Respecto de la segunda parte de la hipótesis, referida a la variedad de fuentes de acuerdo al canal informativo, la información la entrega el cuadro siguiente:

\section{IABLA No. 4}

Variedad de fuentes según canal informativo (porcentajes)

\section{Rutinario}

Informa

Iniciativa medio

Total

Tipo de fuente:

$\begin{array}{llrrr}\text { Gobierno } & 43,6 \% & 7,2 \% & 9,8 \% & 20,8 \% \\ \text { Afiliada } & 43,6 \% & 79,3 \% & 59,0 \% & 59,5 \% \\ \text { Asociada } & 11,9 \% & 7,2 \% & 7,4 \% & 10,1 \% \\ \text { Independiente } & 0,9 \% & 6,3 \% & 23,8 \% & 10,6 \% \\ & 100 \% & 100 \% & 100 \% & 100 \%\end{array}$

Rutinario Informal $\begin{gathered}\text { Iniciativa } \\ \text { medio }\end{gathered} \quad$ Total

Nivel de fuente:

$\begin{array}{llrrr}\text { Ejecutivo } & 71,8 \% & 52,5 \% & 45,0 \% & 56,5 \% \\ \text { Vocero } & 1,7 \% & 6,2 \% & 7,4 \% & 5,1 \% \\ \text { Funcionario } & 6,0 \% & 10,3 \% & 30,3 \% & 16,1 \% \\ \text { No especif, } & 20,5 \% & 31,0 \% & 17,3 \% & 22,3 \% \\ & & & & \\ & 100 \% & 100 \% & 100 \% & 100 \%\end{array}$

Los porcentajes totales demuestran que, en general, hay gran concentración tanto en el tipo como en el nivel de las fuentes. Para efectos del análisis es preciso distinguir entre ambas clasificaciones, puesto que el canal informativo actúa de manera diferente en ambos casos.

En lo que respecta al tipo de fuente, los resultados se oponen -en parte- a lo esperado en la hipótesis. Se observa que el canal rutinario no es el que ofrece la mayor concentración. Se recurre tanto a fuentes de gobierno como a afiliadas, y a una que otra asociada ${ }^{15}$. Esto se explica por el origen de las noticias rutinarias, que no sólo responden a iniciativas del gobierno, sino también de privados. El gran defecto, que trabaja en favor de la hipótesis, es el escaso porcentaje de fuentes independientes, que no por tener ese carácter tienen menos autoridad o son menos válidas y legítimas. Este hecho es significativo y se ajusta con la hipótesis: las fuentes independientes ofrecen una variedad de información mucho mayor que las rutinarias, y esto se comprueba con la numerosa presencia de este tipo de fuentes en las notas de iniciativa del medio. El caso de estas noticias representa la mayor diversificación, donde las fuentes inde-

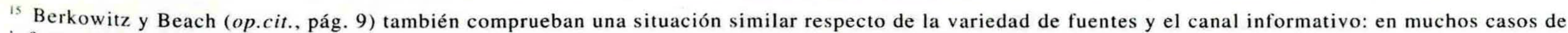

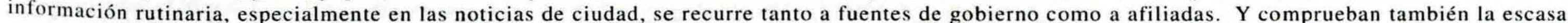
presencia de fuentes independientes.
} 
pendientes ocupan un cuarto del total, aunque se mantiene el predominio de las fuentes afiliadas.

Respecto del nivel de la fuente, los resultados generales también demuestran una alta concentración, especialmente en torno a la fuente ejecutiva. Sin embargo, aquí sí se demuestra la hipótesis. Las noticias rutinarias recurren con excesiva frecuencia a ese tipo de fuentes, y el segundo mayor porcentaje corresponde a las fuentes no especificadas, lo que no representa un buen indicio, en tanto son un tipo de información atribuida a una fuente casi desconocida. El canal de iniciativa del medio obtuvo un porcentaje más cercano a una diversificación óptima, aunque con síntomas de concentración.

En general, esta tabla es más efectiva si se la analiza desde una perspectiva global: no sólo el periodismo económico rutinario esta pecando de poca variedad en las fuentes, sino todo tipo de noticias económicas. Cabe destacar, eso sí, que son las noticias de iniciativa del medio las que más se alejan de la tendencia a la concentración.

La tabla No.5 muestra cómo se relaciona el canal informativo con la calidad de la noticia, acotada por los índices antes descritos. Aquí se pone a prueba la tercera parte de la hipótesis:

\section{IABLA No.5}

Calidad de la noticia según canal informativo (porcentaje de noticias que incluyen el indicador señalado)

\section{Rutinario}

$\mathrm{N}^{\circ}$

CON:

- Instrucción

- Proceso

- Antecedentes y consecuencias

- Pros y contras

- Intereses act,

$\begin{array}{lccc}\text { utinario } & \text { Informal } & \begin{array}{c}\text { Iniciativa } \\ \text { medio }\end{array} & \text { Total } \\ (74) & (36) & (40) & (150) \\ & & & \\ 6,8 \% & 11,1 \% & 7,5 \% & 8,0 \% \\ 35,1 \% & 52,8 \% & 40,0 \% & 40,7 \% \\ & & & \\ 74,3 \% & 77,8 \% & 75,0 \% & 75,3 \% \\ 33,8 \% & 33,3 \% & 45,0 \% & 36,7 \% \\ 25,7 \% & 30,5 \% & 40,0 \% & 30,7 \% \\ 35,1 \% & 41,1 \% & 41,5 \% & 38,3 \%\end{array}$

(El promedio final revela de alguna manera la presencia general de estos indicadores en las noticias de cada canal. Entrega una idea general de lo que sucede en cada canal)

En casi todos los índices de calidad, y en la tendencia general, las noticias de origen rutinario muestran un porcentaje de calidad más bajo que las otras. Aunque a simple vista la diferencia no parece muy grande, se verá más adelante que sí es relevante (especialmente para los diarios chilenos), ya que son las noticias rutinarias de diarios extranjeros las que más contribuyen a elevar este índice. La prensa nacional en general demostró un promedio sumamente bajo, como se verá en la comprobación de la próxima hipótesis.

\section{Conclusión}

La hipótesis 1 queda demostrada casi en su totalidad: existe una alta dependencia respecto de los canales rutinarios (alcanza a casi un 50\% de las noticias); el canal rutinario demostró tener el promedio más bajo de fuentes por noticia; resultó ser parte de la tendencia a la concentración en cuanto a la variedad de fuentes y comprobó tener índices de calidad de la noticia más bajos que el promedio general.

\section{Comprobación Hipótesis 2}

Para analizar esta hipótesis, se exponen las tablas 6,7 y 8 :

\section{IABLA No.6}

Origen de la noticia según nacionalidad del medio

$\begin{array}{clccl} & \text { Rutinario } & \text { Informal } & \begin{array}{c}\text { Iniciativa } \\ \text { medio }\end{array} & \text { Total } \\ \text { Chileno } & 44 & 12 & 19 & 75 \\ \% & 58,7 \% & 16,0 \% & 25,3 \% & 100 \% \\ \text { Extranjero } & 30 & 24 & 21 & 75 \\ \% & 40,0 \% & 32,0 \% & 28,0 \% & 100 \%\end{array}$


Aquí se demuestra que los medios chilenos recurren con mayor frecuencia que los extranjeros a los canales rutinarios. A su vez, registran el índice menor de noticias con iniciativa del medio. Esto no sería un problema si no repercutiera directamente en la calidad de la noticia, como se aprecia a continuación:

\section{TABLA No.7}

\section{Calidad de la noticia según nacionalidad del medio} (porcentaje de noticias que incluyen el indicador señalado)

\begin{tabular}{|c|c|c|c|}
\hline & Chileno & Extranjero & Total \\
\hline & $\mathrm{N}^{\circ} 75$ & 75 & 150 \\
\hline \multicolumn{4}{|l|}{ CON: } \\
\hline - Instrucción & $5,3 \%$ & $10,7 \%$ & $8,0 \%$ \\
\hline - Proceso & $24,0 \%$ & $57,3 \%$ & $40,7 \%$ \\
\hline \multicolumn{4}{|l|}{ - Antecedentes } \\
\hline y consecuencias & $61,3 \%$ & $89,3 \%$ & $75,3 \%$ \\
\hline - Pros y contras & $21,3 \%$ & $52,0 \%$ & $36,7 \%$ \\
\hline - Intereses act, & $14,7 \%$ & $46,7 \%$ & $30,7 \%$ \\
\hline & $25,3 \%$ & $50,1 \%$ & $38,3 \%$ \\
\hline
\end{tabular}

(El promedio final revela de alguna manera la presencia general de estos indicadores en las noticias según origen del medio. Entrega una idea general de lo que sucede en cada caso).

Lo que esta tabla demuestra es grave: los medios chilenos registran una muy baja calidad noticiosa, según el modelo usado para este estudio, muy ligado al hecho de que son altamente dependientes del canal ruti- nario $^{16}$ de información y de que registran el menor número promedio de fuentes por noticia.

\section{Conclusión}

Lamentablemente, lo que se desprende de la clara comprobación de esta hipótesis es la mala situación en que están nuestros diarios respecto de los extranjeros. La idea no es entrar en detalles, pues el comportamiento no varía considerablemente entre los tres diarios nacionales. Sin embargo, sí varía respecto de la realidad periodística que viven los tres diarios extranjeros.

\section{Comprobación Hipótesis 3}

Las siguientes tablas $(8,9,10,11$ y 12) nos dan una idea de la frecuencia con que se recurre al reporteo adicional, y cómo éste afecta distintas variables:

IABLA No. 8

\section{Reporteo adicional (porcentaje total)}

$$
\text { Con reporteo adicional } \quad 48,7 \%
$$

Sin reporteo adicional

\section{TABLA No.9}

\section{Reporteo adicional según canal informativo} (porcentajes)

$\begin{array}{lcccc} & \text { Rutinario } & \text { Informal } & \begin{array}{c}\text { Iniciativa } \\ \text { medio }\end{array} & \text { Total } \\ \text { CON } & 40,5 \% & 50,0 \% & 62,5 \% & 48,7 \% \\ \text { SIN } & 59,5 \% & 50,0 \% & 37,5 \% & 51,3 \% \\ \text { Total } & 100 \% & 100 \% & 100 \% & 100 \%\end{array}$

${ }^{16}$ Sin olvidar el diario Estrategia, que escapa a la tendencia demostrada por los otros dos medios chilenos, en cuanto al origen de la noticia. 
Estas tablas nuevamente favorecen al canal de información de iniciativa del medio. Si bien el porcentaje general entre las noticias con reporteo adicional y sin él es bastante parejo, las que superan el promedio son las informaciones de iniciativa. Las notas rutinarias están claramente bajo el promedio. Esto es importante porque, como se comprueba a continuación, el reporteo adicional también afecta a la cantidad de fuentes por noticia y, como es ya de suponer, la calidad de la noticia.

\section{TABLA No.10}

\begin{tabular}{lccc}
\multicolumn{4}{c}{ Promedio de fuentes según reporteo adicional } \\
& $\begin{array}{l}\text { Con reporteo } \\
\text { adicional }\end{array}$ & $\begin{array}{c}\text { Sin reporteo } \\
\text { adicional }\end{array}$ & Total \\
Noticias & 73 & 77 & 150 \\
Fuentes & 233 & 103 & 336 \\
& & & \\
Promedio & 3,2 & 1,3 & 2,24
\end{tabular}

TABLA No.11

Promedio de fuentes según canal informativo y reporteo adicional

\section{Rutinario}

Informal

3,6

1,8

2,7 1,3 1,6

\section{Iniciativa medio}

Sin r.a.

4,3
1,0
3,1

3,1
Total

Las tablas No. 10 y 11 dejan en evidencia la influencia del reporteo adicional en la cantidad de fuentes. Es importante hacer notar en el caso de las noticias de origen rutinario que el reporteo adicional aumenta la cantidad promedio de fuentes, lo que sirve para contrarrestar el efecto que tiene este canal sobre la variedad de las fuentes y la calidad de la noticia.
La tabla No.12 muestra la variedad de fuentes según la presencia o ausencia de reporteo adicional:

TABLA No.12

Variedad de fuentes según presencia de reporteo adicional

$\begin{array}{lccc}\text { Tipo de fuente: } & \begin{array}{c}\text { Con reporteo } \\ \text { adicional }\end{array} & \begin{array}{c}\text { Sin reporteo } \\ \text { adicional }\end{array} & \text { Total } \\ \text { Gobierno } & 17,4 \% & 28,7 \% & 20,8 \% \\ \text { Afiliada } & 59,6 \% & 58,4 \% & 59,5 \% \\ \text { Asociada } & 8,5 \% & 10,9 \% & 10,1 \% \\ \text { Independiente } & 14,5 \% & 2,0 \% & 10,6 \% \\ & 100 \% & 100 \% & 100 \% \\ \text { Nivel de la fuente: } & & & \\ \text { Ejecutivo } & 56,6 \% & 58,4 \% & 56,5 \% \\ \text { Vocero } & 5,1 \% & 5,0 \% & 5,1 \% \\ \text { Funcionario } & 20,9 \% & 5,0 \% & 16,1 \% \\ \text { No especif, } & 17,4 \% & 31,6 \% & 22,3 \% \\ & 100 \% & 100 \% & 100 \%\end{array}$

Existe, como ya se comprobó en la tabla No.4, una tendencia a la concentración en el nivel y en el tipo de fuente.

En el caso del tipo de fuente, y muy en función de la hipótesis 3 , el reporteo adicional constituye una clara ruptura en este sentido, registrando mayor variedad de fuentes. Nuevamente cabe destacar que toma especial relevancia esta diferencia en el caso de las independientes, que son casi inexistentes en las noticias sin reporteo adicional. Pero se mantiene el predominio de las fuentes afiliadas en ambos casos.

En lo que respecta al nivel de la fuente, si bien no hay mucha diferencia respecto del promedio general en las notas con reporteo adicional, se benefician del hecho que no recurren con tanta frecuencia a fuentes no especificadas. Este recurso es más común en las notas sin reporteo adicional. 
La tabla No.13 muestra cómo se relaciona este esfuerzo reporteril con la calidad de la noticia:

\section{IABLA No. 13}

Calidad de la noticia según reporteo adicional (porcentaje de noticias que incluyen el indicador señalado)

$\mathrm{N}^{\mathrm{O}}$ adicional

73

$15,1 \%$

$60,3 \%$

$91,2 \%$

$68,5 \%$

$58,8 \%$

$$
58,9 \%
$$

Sin reporteo
adicional
77

Total

- Instrucción

-Antecedentes

y consecuencias

- Pros y contras

- Intereses act.
$1,3 \%$

$22,1 \%$

$8,0 \%$

$40,7 \%$
(El promedio final revela de alguna manera la presencia general de estos indicadores en las noticias según la presencia de reporteo adicional. Entrega una idea general de lo que sucede en cada caso).

Nuevamente, la tabla de indicadores de calidad muestra una realidad muy clara. El reporteo adicional es determinante en el contenido de las noticias que publican los medios. El promedio final de presencia de los indicadores es cercano al $60 \%$ en las notas con esfuerzo de reporteo, lo que refleja que es el reporteo adicional el que puede ofrecer una mejor información, casi independientemente de los otros factores.

Este es otro antecedente que apoya la idea de que el reporteo adicional sirve para dar solución a los problemas ligados a las noticias de origen rutinario.

\section{Conclusión}

La hipótesis se mostró evidente: el reporteo adicional contribuye no sólo a aumentar el promedio de fuentes por noticias, sino también a aumentar la presencia de fuentes independientes, y a mejorar notoriamente la calidad de las noticias. Sin embargo, como se comprueba a continuación, este elemento no es muy común entre los medios chilenos.

\section{Comprobación Hipótesis 4}

Para comprobar esta hipótesis, basta con mirar la siguiente tabla:

\begin{tabular}{cccc}
\multicolumn{4}{c}{ IABLA No.14 } \\
Reporteo adicional según nacionalidad \\
$\begin{array}{cccc}\text { (porcentajes) } \\
\text { Con reporteo } \\
\text { adicional }\end{array}$ & $\begin{array}{c}\text { Sin reporteo } \\
\text { adicional }\end{array}$ & Total \\
Chileno & $28,0 \%$ & $72,0 \%$ & $100 \%$ \\
Extranjero & $69,3 \%$ & $30,7 \%$ & $100 \%$
\end{tabular}

Nuevamente se comprueba que el periodismo nacional se queda atrás: los porcentajes casi se invierten al compararse los resultados según el origen del medio ${ }^{17}$. Esto explica, coincide y tiene relación con resultados

\footnotetext{
17 Nuevamente corresponde destacar el caso de Estrategia., que es el medio chileno que más reporteo adicional presenta, como se comprueba a continuación: TABLA No.15.1 Reporteo adicional por diario (porcentajes)

Estrat. Mercurio Diario Ambito Wall Street F.Times

$\begin{array}{lllllll}\text { CON } & 44 \% & 16 \% & 28 \% & 64 \% & 88 \% & 56 \% \\ \text { SIN } & 56 \% & 84 \% & 72 \% & 36 \% & 12 \% & 44 \%\end{array}$

$72 \%-36 \% \quad 12 \% \quad 44 \%$
} 
demostrados en tablas anteriores: la alta dependencia respecto del canal rutinario en los diarios nacionales (tabla No.6), el bajo número promedio de fuentes por noticias (tabla No.1) y la mala calidad de la noticia de nuestro periodismo económico (tabla No.7).

\section{Conclusión:}

La hipótesis 4 se comprueba totalmente: con sólo ver cuánto es el porcentaje de reporteo adicional en nuestro país, se explica también el bajo número de fuentes por noticias y la mala calidad de ellas.

\section{Conclusiones generales}

Es necesario aclarar que este estudio está hecho sobre la base de 150 noticias, durante cinco semanas, en seis medios. Es una muestra pequeña, de la que no se puede -y no se pretende- extraer conclusiones generalizadoras y absolutas. Su utilidad radica en que arroja algunas luces sobre lo que sucede en el periodismo económico chileno, más allá de lo que puede observarse con la simple lectura de los diarios.

Se constata la existencia de un primer problema en la muestra: la alta dependencia de noticias rutinarias, especialmente entre los medios chilenos. Podría no ser un problema en sí, pero está directamente relacionado con la presencia de pocas fuentes en ese tipo de noticias, lo que, a su vez, se traduce en noticias de baja calidad. Las fuentes, en este tipo de noticias, no sólo son escasas, sino poco variadas, con muy baja participación de fuentes independientes. Es posible que esto se deba al sesgo intencional con que fue hecha la muestra, escogiendo sólo las noticias principales publicadas por cada medio. Esto implicaría que, de existir una cantidad importante de iniciativa, ella no se lleva a efecto en los temas que el propio diario considera más importantes.

Este problema se ve agravado por la ausencia de iniciativa de los medios chilenos, dentro de lo analizado en la muestra. No sólo en cuanto a la búsqueda de noticias, sino también en la búsqueda de nuevas fuentes y de profundización de los temas publicados. Lamentablemente, los efectos son una mala calidad de la noticia, que es el producto que lee el público general.

Pero no sólo se constatan cosas negativas. Quedó demostrado, a través del estudio, que el reporteo adicional ofrece una buena posibilidad para superar los problemas asociados a las noticias de origen rutinario. Aunque los medios chilenos dependen en gran medida de este tipo de noticias, ello no tiene por qué obligarlos a entregar noticias de mala calidad.

El planteamiento de Berkowitz y Beach se comprueba: existe una clara y directa influencia entre las fuentes y el contenido de las noticias. Si el periodismo chileno recurre a pocas fuentes, y si ellas están altamente concentradas, difícilmente va a poder entregar una buena información al público.

\section{Bibliografía}

-Berkowitz, Dan y Douglas W. Beach: "News Sources and News Context: The Effect of Routine News, Conflict and Proximity", Journalism Quarterly, Vol. 70, No.1, Primavera 1993.

-Brown, Jane Delano, Carl R. Bybee, Stanley T. Wearden y

Dulcie Murdock Straughan: "Invisible Power: Newspaper News Sources and the Limits of Diversity", Journalism Quarterly, Vol. 64, 1987.

-Coulson, David: "Impact of Ownership on Newspaper Quality”, Journalism Quarterly, Vol.71, No.2, Verano 1994.

-Hofsetter, Richard y David Dozier: "Useful News, Sensational News: Quality, Sensationalism and Local TV News", Journalism Quarterly, Vol.63, No.4, Invierno 1986.

-Riffe, Daniel, Charles F. Aust y Stephen R. Lacy: "The Effectiveness of Random, Consecutive Day and Constructed Week in Newspaper Content Analysis", Journalism Quarterly, Vol.70, No.1, Primavera 1993.

-Sigal, Leon V.: Reporters and Officials: The Organization and Politics of Newsmaking, D.C. Heath \& Company, Lexington, Mass., 1973.

- Soloski, John: "Sources and Channels of Local News", Journalism Quarterly, Vol.66, 1989. 